\title{
A New Multi-Graft Implanter System
}

\author{
Jean M. Devroye, MD, FISHRS I Brussels, Belgium I jeandevroye@aol.com \\ Disclosure: Dr. Devroye is owner of Devroye Instruments and proprietor of the Multi-Graft Implanter.
}

\section{INTRODUCTION}

Sharp implanters have been used for a long time, especially in Korea where they were invented. The implanter's placing is essentially a stick-and-place technique with the incision and placement being made in the same sequence. ${ }^{1}$ In the past few years, another derivative technique, dull needle implanter (DNI), has appeared. It's difficult to know who invented it. Speranzini, who wrote three important articles in this publication in 2016 and 2017, contributed largely to the popularization of the DNI. ${ }^{2-4}$

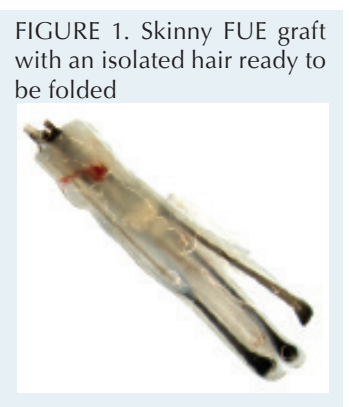

FIGURE 2. J Hair

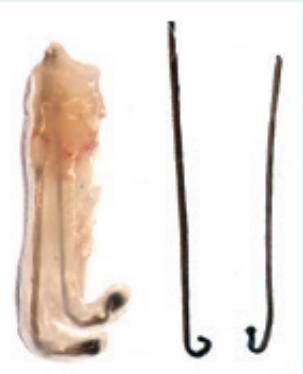

The DNI technique has indisputable advantages:

- Follicles are protected by the implanter's wall. There is minimal trauma during the graft's insertion, which is crucial especially when the FUE grafts are poor in surrounding tissue and present with significant splay (Figure 1). With forceps it is possible to create a "J hair" deformity (Figure 2), while with implanters, the rate of this injury decreases dramatically. It's common to believe J hairs can decrease the rate of hair growth by $30 \% .{ }^{5}$

- It's also obvious dull needle implanters are safer because you are no longer transferring a sharp instrument from loader to user and this reduces the chance of a puncture wound for your team.

- The pre-made incisions are created by blades cut to size. These blades and their incisions are very thin compared to the holes made by a needle implanter. The length of the incision made by the blade is $0.1 \mathrm{~mm}$ smaller than the incision made by the implanter needle. It is easy, therefore, to build a very precise pattern using the combination.

- Finally, it's possible to delegate a large portion of the placing to the assistants; something impossible to do with the sharp implanters.

\section{Personal experience}

I'm always looking for efficiency, trying to optimize the team's work and ergonomics. For the past couple of years, I began to use only implanters with all FUE grafts. The FUT placement is still manual but I hope my team will be convinced, when performing FUT, to switch to the DNI technique soon.

Over the past few months, we tried using the Lion and Hwang implanters, which were initially sharp, but we transformed them into dull implanters. We still seldom used them.

The main criticisms were the following:

- The need for many assistants; for two placers, we needed four loaders.
- The incessant ballet of the implanters causes fatigue and musculoskeletal injuries.

- The implanters' disassembly and cleaning is also very time consuming.

Although some are highly trained in implanter placement $(1,500$ grafts placed per hour $){ }^{6}$ for the majority of us, the placing with traditional implanters is still long, difficult, and tiring.

\section{A NEW IMPLANTING METHOD}

I often wondered how I could improve the implantation technique. My goal was essentially to reduce the number of movements and simplify the whole system. I realized we would need a multi-graft implanter system.

In 1998, 20 years ago, Rassman and Bernstein, always on the innovation front, wrote an article about their carousel, ${ }^{6}$ which unfortunately was not fully developed. To this day, some surgeons in the United States and Korea are still searching for a semi-automatic system around the principle of a revolving cylinder.,

A multi-graft placing assist device developed in 1994, the Banuchi Graft Dispenser, is an elongated hollow tube having both proximal and distal ends open to facilitate the graft loading and placing processes. ${ }^{9}$

I also tried Erdogan's "KEEP" implanter (Figure 3). ${ }^{10}$ This idea was patented in 2004 by Dr. Sanjiv Vasa. The loading is made on the side of the needle, but more important, the graft is gently slid into a pre-made incision with angled forceps. It worked very well. However, the KEEP implanter has three disadvantages: it

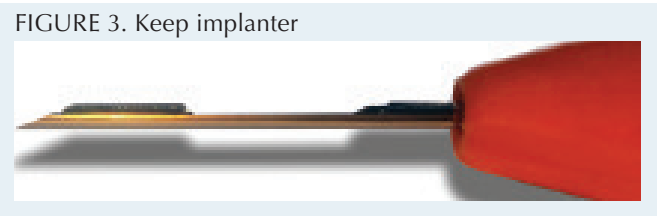

holds only one graft at a time, it is very fragile, and it is very difficult to manage the orientation of the hair's curve.

The orientation of the natural curve of the hair is crucial, especially in the hairline, the temples, and the eyebrow. I always ask my placers to be aware of and maintain the natural curve to give the graft the correct orientation (Figure 4).

I'm a big fan of the adage "less is more" adopted by the architect Ludwig Mies van der Rohe, father of the modern skyscraper. Consequently, I decided to try to create an implanter as simple as possible composed of a long needle with a long slit (Figure 5). I chose the best stainless steel to be strong and removed any sharpness.

All the edges are blunted. I use mainly the $0.7,0.8$, and 
A close FIGURE 7. Implanter tip can be used to adjust graft placement.

$1.0 \mathrm{~mm}$ diameter implanters, though sometimes I use 0.6, 0.9 , and $1.1 \mathrm{~mm}$ implanters. The needle is attached to a Luer Lock Tip in stainless steel.

The grafts are loaded, as with other implanters, from the tip (Figure 6). The grafts are gently slid into the lumen of the implanter. They are moved from the tip to the top. Special attention is taken to give good orientation to the hair curve.

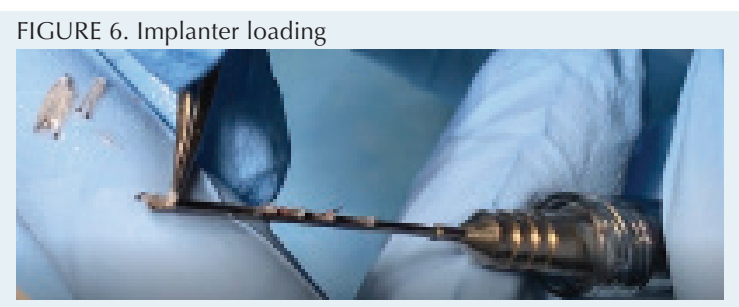

In contrast to the traditional implanter, the curve has to be directed face down. The reason is that the implanter is used with the slit placed upward. The number of grafts loaded varies from 5 to 8 , rarely more. The needle can receive 10 grafts maximum.

The loading is faster than with classical implanters. Less movement means less loss of time.

The implanter doesn't move, the grafts placed near the implanter tip, on the finger, are engaged in the lumen very quickly. The beauty of the system is that now, a placer needs only one loader instead of the two required with the classical DNI technique. The placing is also faster. As you can see on the video I made on YouTube, the implanter tip could be used to adjust the graft's placement (Figure 7). ${ }^{11}$

Multiple loaded implanters can be held and soaked in a chilled or room-temperature storage solution. Ten implanters loaded represents 50-70 grafts ready to place.

With this new implanter, it's also possible to use the traditional stick-and-place technique with two people: one makes the slit and the other places the graft. observation of some grafts removed after placing didn't show any added injuries. In a 2016

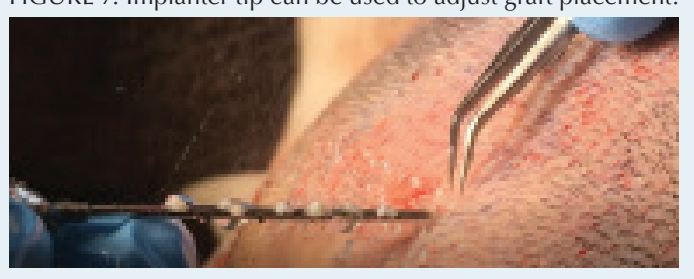

article, von

Albertini demonstrated the absence of grafts suffering from loading and placing injuries in his article. ${ }^{12}$

\section{References}

1. Choi, Y.C., and J.C. Kim. Single hair transplantation using the Choi hair transplanter. Dermatol Surg. 1992; 18:945-948.

2. Speranzini, M. FUE graft placement with dull needle implanters into premade sites. Hair Transplant Forum Int'l. 2016; 26(2):49, 53-56.

3. Speranzini, M. CyberSpace Chat: Use of implanters. Hair Transplant Forum Int'I. 2017; 27(1):22-24.

4. Speranzini, M. Graft placement using the dull needle implanter (DNI) technique. Hair Transplant Forum Int'l. 2017; 27(2):1, 8-12.

5. Cole, J. Project of multigraft implanters. Presented at the October 2017 World Congress of the International Society of Hair Restoration Sugery; Prague.

6. Park, J.H. Novel Implanter Technique That Enables More Than 1,600 Grafts in 1 Hour with Dense Packing. ISHRS Video Library. 2013.

7. Rassman, W., and R. Bernstein. Rapid Fire Hair Implanter Carousel. A new surgical instrument for the automation of hair transplantation. Dermatol Surg. 1998(Jun); 24(6):623-627.

8. Korean Project of Multigraft Implanters. Presented at the October 2017 World Congress of the International Society of Hair Restoration Sugery; Prague.

9. Banuchi, I. Graft Dispenser-Instrument to Facilitate the Hair Transplant Procedure. Presented at the 1997 ISHRS World Congress of the International Society of Hair Restoration Sugery; Barcelona, Spain.

10. Erdogan, K. Keep implanters. YouTube link: www.youtube.com/ watch? $v=$ z9o9S8IrrXA

11. Devroye, J. YouTube link: https://youtu.be/63SAvIBCCws

12. von Albertini, C., and M. von Albertini. Does the use of implanters affect the quality of FUE grafts? Hair Transplant Forum Int'I. 2016; 27(3):96-98. ㅁ

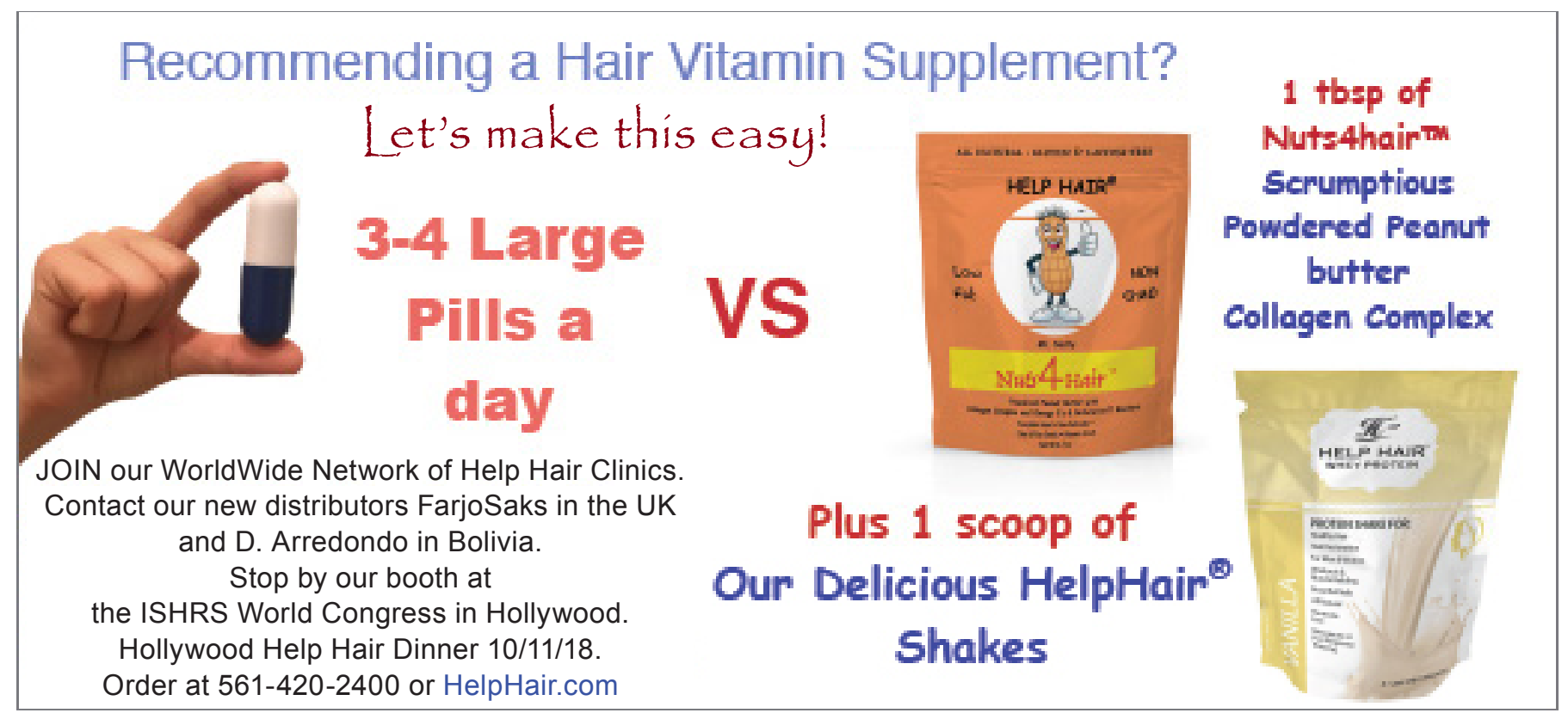

